msh-mss Mathématiques et sciences humaines

168 | Hiver 2004

Les réseaux sociaux

\title{
Introduction au numéro spécial : "Les réseaux sociaux"
}

Foreword. Special issue: "The social networks"

Alain Degenne

\section{(2) OpenEdition}

12 Journals

Édition électronique

URL : http://journals.openedition.org/msh/2964

DOI : $10.4000 / \mathrm{msh} .2964$

ISSN : 1950-6821

Éditeur

Centre d'analyse et de mathématique sociales de l'EHESS

Édition imprimée

Date de publication : 1 décembre 2004

ISSN : 0987-6936

\section{Référence électronique}

Alain Degenne, "Introduction au numéro spécial : "Les réseaux sociaux" », Mathématiques et sciences humaines [En ligne], 168 | Hiver 2004, mis en ligne le 18 mars 2006, consulté le 23 juillet 2020. URL:

http://journals.openedition.org/msh/2964 


\title{
LES RESEAUX SOCIAUX
}

\author{
Alain DEGENNE ${ }^{1}$
}

\section{INTRODUCTION}

Parmi les nombreux foyers de recherche actifs dans le domaine des réseaux sociaux, deux apparaissent particulièrement remarquables :

- l'étude de l'évolution des réseaux de relations, en particulier au moyen de la classe des modèles logits dits $\mathrm{p}^{*}$

- les études autour du petit monde et des réseaux sans dimension.

Les questions sociologiques ou psycho-sociologiques qui les sous-tendent ne sont pas les mêmes et les communautés qui s'y intéressent sont encore assez séparées.

Les amis de mes amis tendent-ils à devenir mes amis ? Autrement dit, si nous observons une communauté d'individus tels que des étudiants qui se trouvent amenés à se côtoyer dans une université ou un internat, doit-on penser que les relations affinitaires qui vont s'instaurer dans le groupe vont tendre à devenir transitives ou pas ? Les jeunes vont-ils se grouper en fonction de similitudes (même sexe, fumeur ou non fumeur, centres d'intérêt communs) ? Dans le même esprit, si l'on observe une communauté à un instant donné et que l'on postule que les relations que l'on y voit constituent une situation de croisière résultant d'un processus déjà ancien, quelles hypothèses peut-on faire sur ce processus ?

Nous sommes conduits à considérer que le réseau que l'on observe est une réalisation d'un réseau aléatoire dans lequel la probabilité d'apparition d'un lien de type $\square$ entre un individu $\mathrm{i}$ et un individu $\mathrm{j}$ dépend des caractéristiques de $\mathrm{i}$ et de $\mathrm{j}$, de l'environnement relationnel de $\mathrm{i}$ et de $\mathrm{j}$, plus généralement d'hypothèses propres à la relation $\square$.

Philippa Pattison cite par exemple un travail qu'elle a réalisé avec Emmanuel Lazega à partir de données recueillies par ce dernier dans un grand cabinet d'avocats d'affaires américains. Cette organisation se compose de 71 personnes qui sont amenées à collaborer pour traiter des dossiers, à se demander des conseils et même à nouer des liens d'amitié. Certains sont des actionnaires de la société, les autres sont seulement des associés. Les auteurs estiment les paramètres d'un modèle qui exprime que la probabilité d'apparition d'un réseau tel que celui qui est observé dépend de la fréquence

\footnotetext{
${ }^{1}$ CNRS, LASMAS, Maison de la recherche en sciences humaines, Université de Caen, Esplanade de la Paix, 14032 Caen, adegenne@wanadoo.fr
} 
de structures locales caractérisant des liens réciproques entre deux personnes ou transitifs dans une triade. En fait, ils calculent les paramètres correspondants à toutes les triades possibles (qui sont au nombre de 15) Ils obtiennent ainsi par exemple un coefficient élevé pour la configuration réciproque concernant la demande d'avis mais aussi pour la configuration transitive. En matière d'amitié, c'est encore la configuration réciproque qui obtient le plus fort coefficient, la transitivité montre un coefficient positif mais nettement plus faible. Ils analysent également les combinaisons entre les trois relations.

Le test d'hypothèses structurelles, telles que celles-ci, sur les réseaux connaît un très grand développement, dans la mesure où l'on cherche à explorer des hypothèses de plus en plus complexes, ce qui pose des problèmes difficiles d'estimation des paramètres. Philippa Pattison présente ici une revue de questions très documentée de ce domaine au développement duquel elle contribue largement.

L'étude de l'évolution des réseaux dans le temps mobilise des modèles assez proches. Tom Snijders a mis au point une méthode d'analyse de ces processus (SIENA) ${ }^{2}$ qu'il présente ici en développant particulièrement une mesure d'évaluation de la qualité d'un modèle. Ces nouveaux outils sont encore peu utilisés par les sociologues français qui disposent rarement de données relationnelles dynamiques.

Faute de disposer d'instruments puissants et faciles à mettre en œuvre pour analyser et représenter de grands réseaux, on s'est pendant longtemps limité à l'examen de réseaux de petite taille correspondant à des petits groupes, des organisations ou des réseaux entre entreprises ou entre pays. La logique de la sociométrie restait très prégnante. Les études des échanges sur internet mais aussi les réseaux de parenté ont conduit à développer des outils d'analyse et de représentation qui peuvent prendre en compte plusieurs milliers de sommets (comme Pajek ${ }^{3}$ ). La possibilité de visualiser de grands réseaux et de voir les résultats de décompositions, d'extractions de sous ensembles a largement changé les pratiques d'analyse et les comportements. L'édition de revues électroniques qui permettent de publier facilement des graphes complexes en couleur joue sans doute également un rôle dans la diffusion de tels travaux.

La mise en œuvre d'une recherche de structures de renchaînement d'alliances telle que celle que présente ici Douglas White n'est rendue possible que grâce à ces nouveaux outils de traitement des grands réseaux. Il faut repérer les cycles dans le graphe de parenté et les classer par types. Même si ces analyses peuvent être programmées en aveugle, le support visuel qui stimule l'intuition facilite grandement le travail. Mais l'intérêt pour les grands réseaux a aussi d'autres raisons.

Dès 1967 l'expérience de Milgram avait attiré l'attention des chercheurs sur l'hypothèse du petit monde. Rappelons que Milgram avait demandé à 296 personnes de faire parvenir un dossier à une personne cible en utilisant uniquement des intermédiaires qui se connaissaient. L'individu-cible était un agent de change résidant à Boston (renseignement connu des participants).

Parmi les 296 individus, 217 ont effectivement expédié le dossier à l'une de leurs connaissances. Au bout du compte, 64 dossiers sont parvenus à l'individu objectif. Le reste correspond à des chaînes rendues incomplètes par l'abandon d'un participant. Parmi les chaînes qui ont abouti 86 \% des participants ont envoyé le dossier à des amis

\footnotetext{
${ }^{2}$ http://stat.gamma.rug.nl/snijders/siena.html

${ }^{3}$ http://vlado.fmf.uni-lj.si/pub/networks/pajek
} 
ou à de simples connaissances et $14 \%$ à des parents. La longueur moyenne des chaînes est de 5,2 intermédiaires.

Ce petit nombre d'intermédiaires suffisant excita la curiosité des chercheurs qui formulèrent l'hypothèse dite du petit monde : avec six intermédiaires, on peut relier deux personnes qui ne se connaissent pas et donc atteindre n'importe qui dans le monde (Six degrees). Le développement d'internet renforça l'intérêt pour cette hypothèse et amena à considérer les structures des réseaux. Barabási rapporte que c'est en observant le Web qu'il constata que plus de $80 \%$ des pages montraient moins de 4 liens alors que moins de $0,01 \%$ en avaient plus de 1000 . En étudiant de plus près cette distribution, il constata que le nombre de liens d'un sommet suivait une distribution puissance. Il existe donc des « hubs» qui sont reliés à de très nombreux sommets, ce qui n'est pas compatible avec le modèle classique de réseau aléatoire, proposé par Renyi et très généralement utilisé, qui pose que la probabilité pour un lien d'exister entre deux sommets suit une loi de Poisson de moyenne $\mathrm{m}$. Cette moyenne étant retenue dans ce cas comme facteur d'échelle du réseau, les réseaux qui suivent une loi puisssance furent qualifiés de «scale free », sans dimension.

D'autres questions sous tendent l'intérêt porté à ces questions, en particulier la diffusion des maladies sexuellement transmissibles : s'il suffit de six intermédiaires pour relier n'importe quelles personnes, si le réseau des échanges sexuels est de ce type, on peut craindre une diffusion très rapide des maladies que l'on ne sait pas traiter. Inversement, s'il existe des « hubs », un tel réseau est peut-être plus facile à attaquer à travers les hubs qu'un réseau ordinaire.

Différents modèles ont été proposés pour engendrer des réseaux sans dimension : créer des courts-circuits dans un réseau ordinaire ou encore définir un processus de génération du réseau fondé sur l'idée que tout nouveau sommet qui se connecte tend à préférer un sommet déjà très populaire. Lin Freeman avait déjà attiré l'attention sur le rôle des individus qui sont des passages obligés entre de nombreuses paires d'individus ; il a proposé une mesure de centralité d'intermédiarité. Dans un réseau sans dimension engendré par un processus de rattachement préférentiel de ce type, il existe des individus dotés d'un fort coefficient d'intermédiarité.

La définition de processus de genèse de réseaux aléatoires sans dimension est devenue une préoccupation importante de certains mathématiciens (Watts, Barabási etc) qui trouvent leur inspiration dans des champs très variés qui vont du métabolisme des cellules aux relations sexuelles, en passant par la structure d'Internet et les citations entre auteurs scientifiques. Pourtant, une base de données du réseau des citations entre les auteurs qui, soit citent Milgram, soit utilisent l'expression petit monde $e^{4}$ montre deux univers encore bien séparés, d'une part les analystes issus de la sociométrie comme Boorman, Burt, Granovetter, Killworth, H. C. White etc, d'autre part des auteurs non sociologues venus plus récemment à l'analyse des réseaux comme Barabási, Dorogovtsev, Newman, Strogatz, Watts etc. Les citations sont nombreuses au sein de chaque groupe et rares entre les deux.

Il est remarquable que l'on retrouve à propos du petit monde une préoccupation classique pour les liens forts et l'articulation entre une propriété micro qui est celle du rôle important des petites structures dans le fonctionnement concret (cercles,

\footnotetext{
${ }^{4}$ Base produite par Institut for Scientific Information of Philadelphia.
} 
organisations locales, niches, mariages préférentiels) avec des caractéristiques globales comme les propriétés de petit monde.

En économie Zimmermann s'intéresse à ces questions [Zimmermann, 2002]. La diffusion des innovations est un autre des sujets qui conduisent à proposer des modèles qui prennent en compte la structure du réseau des échanges et s'éloignent ainsi du modèle trop simple de contagion. Steyer et Zimmermann en proposent ici un exemple.

Les réseaux de parenté sont eux aussi de grands réseaux et leur structure apparaît complexe. La notion de « hub » ne paraît pas rendre compte de cette complexité mais c'est la distribution des sous structures, en particulier des cycles qui permet de les caractériser. À chaque type de cycle est en effet attaché un système de renchaînement d'alliances c'est-à-dire un modèle de mariage préférentiel créant une forme de cohésion dans le groupe en question. Ce type d'observation rapproche les réseaux de parenté de la question du petit monde et Douglas White est l'un des rares auteurs à puiser son inspiration dans les deux domaines. Les outils qu'il propose ici avec ses collègues anthropologues sont très novateurs et apparemment très puissants.

La multiplication des études empiriques correspondant à des domaines divers est un élément important. On peut observer chez certains mathématiciens une tendance à proposer des modèles de génération de réseaux inspirés par des considérations formelles et non fondés par une connaissance empirique des processus concrets. La gamme des modèles susceptibles d'engendrer des réseaux d'apparence complexe est certainement encore très mal connue et l'on ne saurait trop se méfier d'aller vite vers la production d'une économétrie relationnelle mal informée des phénomènes réels.

Il y a là un appel à la contribution des sociologues qui sont restés longtemps enfermés dans une problématique des petits réseaux sociométriques d'avoir à s'intéresser plus nettement à certains aspects de ce que l'on peut appeler le réseau global.

\section{QUELQUES RÉFÉRENCES}

BARABÁSI A.L., BONABEAU E., "Scale-Free Networks", Scientific American, 288, 2003, p. 50-59

KOCHEN M., The Small World, Norwood, Ablex Publishing Corporation, 1988.

LAZEGA E., The Collegial Phenomenon, Oxford, Oxford University Press, 2001.

LAZEGA E., PATTISON P., "Multiplexity, generalized exchange and cooperation in organizations: a case study", Social Networks 21(1), 1999, p. 67-90.

NEWMAN M. E. J., BARABÁSI A. L., WATTS D. J. (eds), The Structure and Dynamics of Complex Networks, Princeton, Princeton University Press, 2003.

PATTISON P., WASSERMAN, S., "Logit models and logistic regressions for social networks. II: Multivariate relations", British journal of Mathematical and Statistical Psychology 52, 1999, p. 169-193.

ROBBINS G., PATTISON P., WASSERMAN S., "Logit models and logistic regressions for social networks. III: Valued relations", Psychometrika 64(3), 1999, p. 371-394. 
SNIJDERS T.A.B, "The Statistical Evaluation of Social Network Dynamics", Sociological Methodology, 2001, p. 361-395.

SNIJDERS T.A.B., "Markov chain Monte Carlo estimation of exponential random graph models", Journal of Social Structure, Vol. 3, n. 2, 2002.

WASSERMAN S., FAUST K., Social Networks Analysis, Cambridge, Cambridge University Press, 1994.

WASSERMAN S., PATTISON P., "Logit models and logistic regressions for social networks. I: An introduction to Markov graphs and p*", Psychometrika 61(3), 1996, p. 401-425.

WATTS D. J., Small Worlds, Princeton, Princeton University Press, 1999.

WATTS D. J., Six Degrees: the Science of a Connected Age, New York, Norton, 2003.

WHITE D. R. (ed.), Complexity 8(1), Special issue, 2002.

ZIMMERMANN J.B., «Des « clusters » aux « small worlds » : une approche en termes de proximités », Géographie, Économie, Société 4, 2002, p. 3-17. 